Research Article

\title{
Epidemiological Features and Predictors of Mortality in Patients with COVID-19 with and without Underlying Hypertension
}

\author{
Leila Moftakhar $\left(\mathbb{D},{ }^{1,2}\right.$ Elahe Piraee $\left(\mathbb{D},{ }^{2}\right.$ Mohammad Mohammadi Abnavi $\left(\mathbb{D},{ }^{2}\right.$ \\ Parisa Moftakhar $\left(\mathbb{0},{ }^{3}\right.$ Habibollah Azarbakhsh $\left(\mathbb{0},{ }^{2}\right.$ and Aliasghar Valipour $\mathbb{1}^{4}$ \\ ${ }^{1}$ Abadan University of Medical Sciences, Abadan, Iran \\ ${ }^{2}$ Student Research Committee, Shiraz University of Medical Sciences, Shiraz, Iran \\ ${ }^{3}$ Department of Clinical Sciences, Faculty of Veterinary Medicine, Shahid Chamran University of Ahvaz, Ahvaz, Iran \\ ${ }^{4}$ Department of Public Health, Abadan Faculty of Medical Sciences, Abadan, Iran
}

Correspondence should be addressed to Habibollah Azarbakhsh; azarbakhshhabibollah@gmail.com

Received 23 April 2021; Revised 3 September 2021; Accepted 12 September 2021; Published 19 October 2021

Academic Editor: Massimo Salvetti

Copyright (๑) 2021 Leila Moftakhar et al. This is an open access article distributed under the Creative Commons Attribution License, which permits unrestricted use, distribution, and reproduction in any medium, provided the original work is properly cited.

\begin{abstract}
Backgrounds. Individuals with hypertension are at higher risk of COVID-19 infection and related mortality. This study was carried out to assess the epidemiological features and predictors of mortality in patients with COVID-19 with hypertension. Methods. In this retrospective study, the epidemiological characteristics of two groups of patients with COVID-19 with hypertension (1927) and without hypertension (39030) were compared. Chi-square test was applied to evaluate the differences between qualitative variables in two study groups. Logistic regression was also used to determine predictors of mortality in patients with COVID-19 and in patients with COVID-19 with hypertension. Results. The prevalence of hypertension in patients with COVID-19 was 4.7\%, and $24.37 \%$ of COVID-19 related deaths occurred in these individuals. The average age of hypertension and nonhypertension patients was 61 and 37 years, respectively. Fever, cough, headache, anorexia, fatigue, and comorbid diseases, such as cardiovascular disease, chronic lung and kidney disease, diabetes, immunodeficiency disease, and thyroid disease, were significantly more frequent in people with hypertension than those without hypertension. The chances of mortality in patient with COVID-19 were 1.8 times higher in individuals with dyspnea, 1.25 in individuals with fever, 1.33 in individuals with cough, 3.6 in patients with hypertension, 2.21 in diabetics, and 2.2 in individuals with cardiovascular disease. Also, individuals with COVID-19 with hypertension that had dyspnea, immunodeficiency, and cardiovascular disease were at higher risk of mortality. Conclusion. Hypertension is a serious threat to patients with COVID-19. Therefore, in order to control these patients more precisely and reduce mortality in them, it is extremely important to develop prevention and treatment strategies.
\end{abstract}

\section{Introduction}

Coronavirus disease (COVID-19) is an infectious disease caused by a newly discovered coronavirus. According to the World Health Organization, COVID-19 disease was first reported in December 2019 in Wuhan, China, and rapidly spread across other parts of the world, causing people various health, economic, social, and political problems around the world [1-3]. According to the latest statistics of the World Health Organization, as of April 21, 2021, there are 144,167,456 cases of infection and about 3 million deaths in the world. Also in Iran, 2.31 million people have been infected and 67,000 deaths have occurred. [4].

Recent studies have shown that COVID-19 patients with older age and comorbidity, such as cardiovascular disease, diabetes, hypertension, or chronic lung disease, are at higher risk of infection with a higher mortality rate compared to the general population $[5,6]$. In the literature, the prevalence of hypertension in people with COVID-19 was $25.4 \%$ in Africa, $31 \%$ in China, $49 \%$ in Italy, $21 \%$ in India, $32 \%$ in Oman, and 
10\% in Iran [7-10]. Therefore, these patients should be given special attention during the epidemic. In another study, the mortality rate of COVID-19 was reported as $6 \%$ in patients with hypertension [11].

Given that hypertension is an important cause of mortality in the world, and a large number of people have the disease in the world, during the COVID-19 pandemic, this vulnerable group of people who are at higher risk of morbidity and mortality from COVID-19 should be given more care. For this purpose, the present study aimed to compare the epidemiological features and predictors of mortality in patients with COVID-19 with hypertension.

\section{Methods}

This retrospective study was conducted on 40957 patients with COVID-19 who were admitted in hospitals under the auspices of Abadan University of Medical Sciences in the southwest of Khuzestan province. The total population of this region was estimated as 627970 people, using the databases of the health centers and the national census conducted in 2020. All patients with COVID-19 from March 1, 2019, to March 1, 2020, were enrolled into this study. Confirmation of definite cases of COVID-19 was performed using nasal and throat swab samples (RT-PCR) or by CT scan imaging. Patients with normal vital signs (blood pressure, respiration rate, and pulse) with saturated oxygen levels above $93 \%$ and mild symptoms were sent to home quarantine and monitored daily by health professionals. Patients were hospitalized with severe symptoms such as chest pain, shortness of breath, and a saturated oxygen level of less than $90 \%$. Patients with saturated oxygen levels between $90 \%$ and $93 \%$ were admitted to hospital or undergoing home quarantine. In the area under study, there were only two hospitals that performed all stages of testing and hospitalization of patients according to the national guidelines of corona management, like other medical centers in the country. Clinical and demographic information of patients, and the final outcome of each patient, was recorded.

Duplicate items were identified and removed based on the National ID card. The subjects were divided into two groups of patients, hypertension ( $n=1927$ patients) and nonhypertension ( $n=39030$ patients). Individuals with systolic blood pressure above $14 \mathrm{mmHg}$ and diastolic above $90 \mathrm{mmHg}$ or individuals who take antihypertensive drugs were identified as patients with hypertension. The history of hypertension and other underlying diseases evaluated in this study is based on patients' self-report and questioning the names of their medications.

Variables used in this study include age, gender, final outcome (e.g., death and recovery) and symptoms (e.g., shortness of breath, fever, cough, taste disturbance, fatigue, muscle pain, sore throat, headache, and comorbidities, such as diabetes, cardiovascular disease, kidney and liver diseases, immune deficiency, chronic lung disease, and thyroid disease).

2.1. Statistical Analysis. We first described qualitative variables with numbers and percentages and quantitative variables with median. Then, we used the Chi-square test for qualitative variables and the $t$-test for the quantitative variable of age to assess the difference between the mean surviving and not surviving in patients with COVID-19 with underlying hypertension. The normality of the age variable was evaluated by KolmogorovSmirnov test.

Finally, we used simple and multiple logistic regression tests to identify the role each of the underlying diseases and other variables on the survival and nonsurvival of patients with COVID-19.

We also used one separately simple and multiple logistic regression tests to identify the factors affecting the mortality of patients with COVID-19 with underlying hypertension.

All variables with P.V less than 0.2 in simple logistic regression test entered to multiple logistic regressions. Significance level was considered 0.05 in all tests. All statistical analyses were performed with SPSS software version 20 and STATA version 14.

\section{Results}

During the study period, 40957 cases of COVID-19 occurred in the cities under the auspices of Abadan University of Medical Sciences in the southwest of Khuzestan province. The median age of the patients was 37 years (IQR 29-50). The majority of patients were male (57.4\%), and 26,252 patients $(64.01 \%)$ had a history of contact with definite or suspected cases of COVID-19. In addition, 1038 patients (2.5\%) died, with $24.37 \%$ of them having occurred in patients with hypertension (Table 1).

The most common symptoms were fever (59.6\%), cough (43.42\%), shortness of breath $(29.86 \%)$, muscle pain $(14.52 \%)$, and sore throat (7.01\%). The most common comorbidities in all study patients include diabetes $(6.94 \%)$, CVDs (4.88\%), chronic lung disease and allergies $(2.41 \%)$, chronic kidney disease (1.43\%), and immunodeficiency diseases (0.95\%) (Table 1$)$.

Patients with hypertension accounted for $4.7 \%$ of the total study population. Besides, $59.5 \%$ of patients with hypertension were female, and $71.35 \%(n=1375)$ had a history of contact with definite or suspected cases of COVID-19. Patients with hypertension were older compared to patients without hypertension. The median age of hypertensive patients was 61 years (IQR: 70-50) versus 37 years (IQR: 50-29) in nonhypertensive patients. Furthermore, prevalence of comorbidities was shown to be higher in hypertensive patients, as diabetes (828 (43\%)) vs. 2014 (5.16), chronic lung disease $(87(4.5 \%)$ vs. $901(2.3 \%))$, chronic kidney disease $(124(6.4 \%)$ vs. $464(1.19 \%)$, systemic disease Immunity $(59(3.06 \%)$ vs. $330(0.84 \%))$, thyroid disease (101 (5.24\%) vs. $74(0.19))$, and CVDs (421 (21.84\%) vs. 1577 $(4.04 \%))$.

Fever, cough, headache, anorexia, and fatigue were significantly higher in hypertensive patients than in nonhypertensive patients. A loss of sense of taste and smell in patients with normal blood pressure was significantly greater in patients with hypertension (Table 1). 
TABLE 1: The characteristics of hypertensive and nonhypertensive patients with COVID-19.

\begin{tabular}{|c|c|c|c|c|}
\hline Variable & $\begin{array}{l}\text { Total }(n=40957) \\
\text { Number }(\%)\end{array}$ & $\begin{array}{c}\text { Hypertension }(n=1927) \\
\text { Number }(\%)\end{array}$ & $\begin{array}{c}\text { Nonhypertension }(n=39030) \\
\text { Number }(\%)\end{array}$ & $P$ value \\
\hline Age, median (IQR) & $37(29-50)$ & $37(29-50)$ & $61(50-70)$ & $<0.001$ \\
\hline \multicolumn{5}{|l|}{ Sex } \\
\hline Male & $23521(57.42)$ & $781(40.50)$ & $22740(58.26)$ & \multirow{2}{*}{$<0.003$} \\
\hline Female & $17436(42.58)$ & $1146(59.50)$ & $16290(41.74)$ & \\
\hline No-sign & $1753(5.50)$ & $94(4.90)$ & $1659(4.25)$ & 0.130 \\
\hline Fever & $20717(50.58)$ & $1163(60.35)$ & 19554 (50.09) & $<0.001$ \\
\hline Cough & $17787(43.43)$ & $1032(53.55)$ & $16755(42.92)$ & $<0.007$ \\
\hline Dyspnea & $12231(29.86)$ & $782(40.58)$ & 11449 (29.39) & 0.100 \\
\hline Muscular pain & $5948(14.52)$ & $391(20.29)$ & $5557(14.23)$ & 0.100 \\
\hline Sore throat & $2872(7.01)$ & $187(9.70)$ & $2685(6.88)$ & 0.350 \\
\hline Anorexia & $273(0.57)$ & $59(3.10)$ & $214(0.55)$ & $<0.001$ \\
\hline Headache & $1263(3.08)$ & $170(8.82)$ & $1093(2.80)$ & $<0.001$ \\
\hline Fatigue & $313(0.76)$ & $92(4.77)$ & $221(0.56)$ & 0.001 \\
\hline Decreased sense of smell, taste & $2176(5.31)$ & $55(2.85)$ & $2121(5.43)$ & $<0.001$ \\
\hline Hypoxia & $2873(7.01)$ & $194(10.07)$ & $2679(6.86)$ & 0.136 \\
\hline \multicolumn{5}{|l|}{ Comorbidities } \\
\hline Cardiovascular & $1998(4.88)$ & $421(21.84)$ & $1577(4.04)$ & $<0.001$ \\
\hline Diabetes & $2842(6.94)$ & $828(43)$ & $2014(5.16)$ & $<0.001$ \\
\hline Immunodeficiency & $389(0.95)$ & $59(3.06)$ & $330(0.84)$ & $<0.001$ \\
\hline Liver & $101(0.25)$ & $10(0.52)$ & $91(0.23)$ & 0.090 \\
\hline Thyroid & $175(0.43)$ & $101(5.24)$ & $74(0.19)$ & 0.001 \\
\hline Chronic kidney disease & $588(1.43)$ & $124(6.43)$ & $464(1.19)$ & $<0.001$ \\
\hline Chronic pulmonary disease & $988(2.41)$ & $87(4.50)$ & $901(2.30)$ & $<0.001$ \\
\hline Hospitalization history in ICU & $102(0.25)$ & $29(1.50)$ & $73(0.19)$ & $<0.001$ \\
\hline Exposure to disease & $26252(64.10)$ & $1375(71.35)$ & $24877(63.74)$ & $<0.001$ \\
\hline Mortality & $925(2.26)$ & $253(13.13)$ & $672(1.72)$ & $<0.001$ \\
\hline
\end{tabular}

The age of patients with COVID-19 with hypertension who died was significantly higher than the age of those who survived (the median age of 68 years (IQR 60-78) vs. 60 years (IQR 49-69)). Fever, cough, and shortness of breath as well as comorbidities, including heart disease, diabetes, and immunodeficiency, as well as history of contact with affected people and hospitalization in the intensive care unit, were significantly higher in those who died than the survivors (Table 2).

The results of logistic regression to determine the predictors of death in all patients with COVID-19 in our study showed that the chance of death is 1.8 times higher in people with dyspnea $(\mathrm{OR}=1.82,95 \% \mathrm{CI}$ : 1.086-2.206), 1.2 times in people with fever ( $\mathrm{OR}=1.25,95 \% \mathrm{CI}$ : 1.019-1.538), 1.3 times in people with cough $(\mathrm{OR}=1.33,95 \% \mathrm{CI}$ : $1.101-1.615), 3.6$ times in people with hypertension $(\mathrm{OR}=3.636,95 \% \mathrm{CI}$ : 2.977-4.440), 2.2 times in people with diabetes $(\mathrm{OR}=2.215$, 95\%CI: $1.832-2.676)$, and 2.2 times in people with cardiovascular disease $(\mathrm{OR}=2.237,95 \% \mathrm{CI}: 1.823-2.745)$ higher than people without theses sign and disease. Also, the role of age was borderline (OR=1.002, 95\%CI: 1.001-1.003). (Table 3).

The results of logistic regression to determine the predictors of death in all patients with COVID-19 with hypertension showed that the chance of death is 2.04 time in people with dyspnea $(\mathrm{OR}=2.046,95 \% \mathrm{CI}$ : 1.506-2.779), 2.7 times in people with immunodeficiency $(\mathrm{OR}=2.709,95 \% \mathrm{CI}$ : 1.424-5.152), and 1.4 times in people with Cardiovascular disease (OR $=1.417,95 \% \mathrm{CI}: 1.011-1.986)$ higher than people without theses sign and disease (Table 4).

\section{Discussion}

The present study was performed on 40957 patients with COVID-19 in the southern region of Iran and aimed to compare the clinical features and the rate of mortality among COVID-19 patients with hypertension compared with those patients with normal hypertension. COVID-19 is a highly contagious disease that has spread rapidly around the world [12]. Several studies have shown that although COVID-19 can affect all healthy people of any age, older people and people with chronic disease are at higher risk, and many studies have shown that patients with hypertension are at higher risk for COVID-19 [13-18]. There are also more complications from COVID-19 and hospitalization in the intensive care unit in patient with hypertension [17-19].

In our study, out of the 40,957 surveyed people, 1927 (4.7\%) had hypertension. Other studies have reported hypertension as one of the most common chronic diseases in patients with COVID-19 [12, 20-22]. Peng and colleagues reported the prevalence of hypertension among COVID-19 patients as $31 \%$ [17]. This rate reported $30 \%$ by Zhou and colleagues [12], and $15 \%$ by Guan and colleagues [23], while China Centers for Disease Control and prevention revealed this as $13 \%$ [24]. In other studies, the prevalence of hypertension in COVID-19 patients has been reported differently as $30.7 \%$ [25], 30\% [26], and 31.8\% [27]. It has been estimated in many studies that people with hypertension make up about one-third of COVID-19 patients, which was 
TABLE 2: The baseline characteristics of survivors and nonsurvivors in patients with COVID-19 with hypertension disease.

\begin{tabular}{|c|c|c|c|c|}
\hline Variable & $\begin{array}{c}\text { Total }(n=1927) \\
\text { Number }(\%)\end{array}$ & $\begin{array}{c}\text { Survivors }(n=1674 \\
\text { Number }(\%)\end{array}$ & $\begin{array}{c}\text { Nonsurvivors }(n=253) \\
\text { Number }(\%)\end{array}$ & $P$ value \\
\hline Age, median (IQR) & $61(50-70)$ & $60(49-69)$ & $68(60-78)$ & $<0.001$ \\
\hline $\begin{array}{l}\text { Gender } \\
\text { Male } \\
\text { Female }\end{array}$ & $\begin{array}{l}781(40.50) \\
1146(59.5)\end{array}$ & $\begin{array}{l}678(40.50) \\
996(59.50)\end{array}$ & $\begin{array}{l}103(40.71) \\
150(59.29)\end{array}$ & 0.980 \\
\hline \multicolumn{5}{|l|}{ Symptoms } \\
\hline Fever & $1156(59.99)$ & $977(58.36)$ & $179(70.75)$ & 0.001 \\
\hline Cough & $1028(53.35)$ & $867(51.80)$ & $161(63.64)$ & 0.002 \\
\hline Dyspnea & $781(40.53)$ & $637(38.05)$ & $144(56.92)$ & $<0.001$ \\
\hline Muscular pain & $388(20.13)$ & $341(20.37)$ & $47(18.58)$ & 0.390 \\
\hline Sore throat & $185(9.60)$ & $160(9.56)$ & $25(9.88)$ & 0.970 \\
\hline Disguise & $54(2.80)$ & $54(3.22)$ & $0(0.00)$ & 0.030 \\
\hline Headache & $168(8.72)$ & $152(9.08)$ & $16(6.32)$ & 0.120 \\
\hline Cardiovascular & $416(21.59)$ & $338(20.20)$ & $78(30.80)$ & 0.001 \\
\hline Diabetes & $822(42.66)$ & $689(41.16)$ & $124(49)$ & 0.04 \\
\hline Immunodeficiency & $58(3.01)$ & $43(2.57)$ & $15(5.93)$ & 0.004 \\
\hline Chronic pulmonary disease & $87(4.51)$ & $71(4.24)$ & $16(6.32)$ & 0.140 \\
\hline Chronic liver, kidney disease & $123(6.38)$ & $105(6.27)$ & $18(7.11)$ & 0.640 \\
\hline Exposure to disease & $1357(78.42)$ & $1195(71.38)$ & $162(64.03)$ & 0.010 \\
\hline Hospitalization history in ICU & $29(1.50)$ & $21(1.25)$ & $8(3.16)$ & 0.020 \\
\hline
\end{tabular}

TABLE 3: Predictor variables of mortality in patient COVID-19 based on the results of multiple logistic regression.

\begin{tabular}{lcc}
\hline Predictors & Odds ratio $(95 \% \mathrm{CI})$ \\
\hline Age & $1.002(1.001-1.003)$ & $P$ \\
Dyspnea & Reference & $<0.001$ \\
No & $1.824(1.086-2.206)$ \\
Yes & Reference \\
Fever & $1.252(1.019-1.538)$ \\
No & Reference \\
Yes & $1.333(1.101-1.615)$ \\
Cough & \\
No & Reference \\
Yes & $3.636(2.977-4.440)$ \\
Hypertension & Reference \\
No & \\
Yes & $2.215(1.832-2.676)$ \\
Diabetes & Reference \\
No & 0.033 \\
Yes & $2.237(1.823-2.745)$ \\
Cardiovascular disease & $<0.003$ \\
No & $<0.001$ \\
Yes & $<0.001$ \\
\hline
\end{tabular}

shown as about $4.7 \%$ in our study. This difference may be due to the very large sample size of our study. Also, about $60 \%$ of our study participants aged under 40 years old, which usually had a lower prevalence of underlying diseases at this age. However, some studies focused only on severe forms of the disease or only hospitalized patients in intensive care units and have shown that these people are also more likely to suffer from underlying diseases, such as hypertension.

Many studies have shown that people with hypertension tend to have more severe forms of COVID-19 and are more likely to be hospitalized in the ICU than people without high blood pressure, with a higher rate of mortality $[28,29]$. In our study, the rate of mortality in people with hypertension compared to people with normal blood pressure was $1.5 \%$ vs.
0.19\%. In general, about a quarter $(24.3 \%)$ of COVID-19 related deaths occurred in hypertensive patients. A systematic review study also found that people with hypertension were 2.2 times more likely to die. [30]. In Spain, the odds of mortality were estimated as 4.7 times [31]. Yang et al. showed the rate of death due to COVID-19 in hypertensive patients as $17.7 \%$ [18]. Also, Zhou and colleagues showed that the crude mortality rate was $19.4 \%$ in the hypertensive group vs. $2.4 \%$ in nonhypertensive group of patients [32], whereas this was reported as $37.1 \%$ vs. $8.7 \%$ in Cheng's study [18].

Also, the rate of ICU stay in patients with hypertension was $13.13 \%$ versus $1.7 \%$ in people without hypertension. A systematic review study found that patients with hypertension were 2.1 times more likely to be admitted to the ICU 
TABLE 4: Predictor variables of mortality in COVID-19 patients with hypertension based on the results of multiple logistic regression.

\begin{tabular}{lll}
\hline Predictors & Odds ratio (95\%CI) & \\
\hline & & \\
Dyspnea & & Reference \\
$\quad$ No & $2.046(1.506-2.779)$ & \\
$\quad$ Yes & & \\
Immunodeficiency & $2.709(1.424-5.152)$ & Reference \\
$\quad$ No & & \\
$\quad$ Yes & $1.417(1.011-1.986)$ & Reference \\
Cardiovascular disease & & $<0.001$ \\
$\quad$ No & & \\
$\quad$ Yes & & 0.043 \\
\hline
\end{tabular}

and 1.7 times more likely to have more severe disease [30]. In a meta-analysis study, the odds of admitted to the ICU were estimated to be 2.5 [33]. Also, this rate in people with high blood pressure compared to people with normal blood pressure was estimated $18.6 \%$ vs. $8.7 \%$ [27] and $32.4 \%$ vs. $19.1 \%$ [17]. However, Yang and colleagues stated that hypertension was not associated with higher risk of developing severe form of COVID-19 disease [18]. The main reason for the higher mortality rate and hospitalization in the ICU in this group of patients is unknown. However, some studies have suggested the presence of ACE2A enzyme in the body as a possible cause of higher rates of death and ICU stay. This is an internal membrane glycoprotein lung tissue that is found in epithelial cells of the heart, liver, and lungs [34, 35], converts angiotensin 2 to angiotensin 1-7, and increases the anti-inflammatory and antioxidant role of angiotensin 1-7, that leading to vasodilation and lowered blood pressure $[36,37]$. Coronavirus also uses ACE2 as a receptor to bind and enter the host body [7, 38-40], thus increasing the expression of ACE2 gene and increasing the risk of COVID19 infection [41, 42]. ACE2 enzyme is increased in patients with hypertension due to the use of ARBS and ACEIS drugs, which in turn increases the risk of COVID-19 infection. In other studies, hypertension has been suggested to cause pathogenic changes in the cardiovascular system, such as fibrosis and left ventricular hypertrophy, which may increase the odds of getting severe form of the infection due to shortness of breath [43].

The results of our study showed that the chance of death is 2.7 times higher in individuals with impaired immune systems. Impaired immune system is also one of factors associated with the severity of the disease in these people [44]. Some studies have also shown that cytokine storms, such as elevated levels of interleukin-6, interleukin-7, and granulocyte-macrophage cloning replicator, are associated with more severe forms of COVID-19 in people with hypertension [40]. Individuals with hypertension are usually older and often have one or more underlying disease, in which their role in determining the severity of the disease cannot be ignored. In our study, $4.7 \%$ of COVID-19 patients had hypertension that $42.6 \%$ of them had diabetes and $21.5 \%$ had heart disease. Other previous studies have shown that COVID-19 patients with hypertension display higher frequency of different comorbidities [27]. Cheng and et al. stated that $22.9 \%$ of hypertensive patients had diabetes versus $4 \%$ in nonhypertensive [27]. This may be due to the fact that the proportion of patients with heart problems is higher in people with hypertension [45], and many other studies have found that heart disease and diabetes are much higher in COVID-19 patients with hypertension than in nonhypertensive persons. [29, 32]. Therefore, clinical research is needed to differentiate the role of each factor.

According to the results of the present study, patients with COVID-19 who had hypertension were older than those with normal hypertension (61 years vs. 37 years). This median age has been reported in other studies as follows: in China 69 vs. 59 years [46], in a cohort study in China 59 vs. 41 years [29], and in Huang et al.'s study 67 vs. 57 years [40]. This may be due to the fact that blood pressure usually increases in older age.

In our study, about $60 \%$ of COVID-19 cases who had hypertension were males. In another study, it was shown that men were more susceptible to the disease and severe complications, 56.1\% [23] and 69.3\% [40], which, of course, should be noted that that men are more at risk of developing COVID-19 infection than women, because they leave home more frequently to work and do housework.

The results of our study also showed that the symptoms such as cough, headache, anorexia, and fatigue in hypertensive patients were significantly higher than nonhypertensive COVID-19 patients. Also, the chance of mortality in patients with dyspnea was 2 times. In another study, nocturnal sweating and shortness of breath were shown to be more common in people with hypertension pressure than nonhypertensive [32]. These symptoms can result from the more severe and worsening of the condition of hypertensive patients, who are more severely affected by the disease.

Along with all the topics mentioned, it should be noted that other factors such as environmental factors can play a role in the severity and mortality of COVID-19. The results of a meta-analysis conducted in European countries and China showed that the odds of mortality in people with COVID-19 between March to May are 0.98 times higher per day after admission and 0.85 times higher with increase in temperature of $1^{\circ} \mathrm{C}$. Another analysis of the symptoms of 37,184 patients in the UK showed that, during this time, the severity of the disease and the symptoms of the disease decreased [47]. These results indicate the role of environmental factors. Although we did not have data on these factors in our study, we should not ignore their role. 


\section{Limitations}

Our study is one of the first in Iran that examined the clinical features of COVID-19 patients with and without hypertension with a relatively large sample size. But one of the limitations of our study was the lack of access to information about the severity of the disease, including the percentage of saturated oxygen and the degree of fever. Therefore, we could not compare the rate of mortality according to the severity of the disease. However, it should be considered that the laboratory and radiological results of patients were not available for more detailed analysis.

\section{Conclusion}

According to the results of our study, hypertension is a major concern for patients with COVID-19, because it increases the rate of hospitalization and death. There is still a lot unknown about COVID-19 disease, and the link between hypertension and COVID-19 that creates great challenges for management and monitoring of these patients for health policymakers. Therefore, in order to control these patients more precisely and effectively, and to reduce the incidence and morbidity, it is recommended to develop prevention and treatment strategies for this group of people who are a vulnerable group against COVID-19. In addition, patients should take preventive measures, use home quarantine, frequently wash their hands, and use blood pressure monitors and controls at home to reduce the number of visits to medical centers.

\section{Data Availability}

The data for the current study will not be shared publicly.

\section{Conflicts of Interest}

The authors declare that they have no conflicts of interest.

\section{Acknowledgments}

The authors would like to acknowledge the support of the Department of Health of Abadan University of Medical Sciences.

\section{References}

[1] L. Moftakhar, P. Moftakhar, E. Piraee, H Ghaem, A Valipour, and $\mathrm{H}$ Azarbakhsh, "Epidemiological characteristics and outcomes of COVID-19 in diabetic versus non-diabetic patients," International Journal of Diabetes in Developing Countries, vol. 41, no. 3, pp. 1-6, 2021.

[2] L. Moftakhar and M. Seif, "The exponentially increasing rate of patients infected with COVID-19 in Iran," Archives of Iranian Medicine, vol. 23, no. 4, pp. 235-238, 2020.

[3] H. Yi, "2019 novel coronavirus is undergoing active recombination," Clinical Infectious Diseases, vol. 71, no. 15, pp. 884-887, 2020.

[4] https://www.worldometers.info/coronavirus/.

[5] A. K. Singh, R. Gupta, and A. Misra, "Comorbidities in COVID-19: outcomes in hypertensive cohort and controversies with renin angiotensin system blockers," Diabetes \& Metabolic Syndrome: Clinical Research Reviews, vol. 14, no. 4, pp. 283-287, 2020.

[6] C. Mancusi, G. Grassi, G. Grassi et al., "Clinical characteristics and outcomes of patients with COVID-19 infection: the results of the SARS-RAS study of the Italian society of hypertension," High Blood Pressure and Cardiovascular Prevention, vol. 28, no. 1, pp. 5-11, 2021.

[7] T. M. Cook, "The importance of hypertension as a risk factor for severe illness and mortality in COVID-19," Anaesthesia, vol. 75, no. 7, pp. 976-977, 2020.

[8] Y. Gholampour, B. Tehranineshat, H. Najafi, M. Farjam, Z. Rahimi, and M. Bijani, "A study of demographic characteristics, clinical manifestations, radiologic and lab findings of patients hospitalized with COVID-19 in the south of Iran," Journal of Fasa University of Medical Sciences, vol. 10, no. 3, pp. 2456-2465, 2020.

[9] F. Khamis, I. Al-Zakwani, H. Al Naamani et al., "Clinical characteristics and outcomes of the first 63 adult patients hospitalized with COVID-19: an experience from Oman," Journal of Infection and Public Health, vol. 13, no. 7, pp. 906-913, 2020.

[10] J. B. Nachega, D. K. Ishoso, J. O. Otokoye et al., "Clinical characteristics and outcomes of patients hospitalized for COVID-19 in Africa: early insights from the Democratic Republic of the Congo," The American Journal of Tropical Medicine and Hygiene, vol. 103, no. 6, pp. 2419-2428, 2020.

[11] C. E. L. Sisnieguez, W. G. Espeche, and M. R. Salazar, "Arterial hypertension and the risk of severity and mortality of COVID-19," European Respiratory Journal, vol. 55, no. 6, 2020.

[12] F. Zhou, T. Yu, R. Du et al., "Clinical course and risk factors for mortality of adult inpatients with COVID-19 in Wuhan, China: a retrospective cohort study," The Lancet, vol. 395, no. 10229, pp. 1054-1062, 2020.

[13] P. M. De Salazar, R. Niehus, A. Taylor, C. O. F. Buckee, and M. Lipsitch, "Identifying locations with possible undetected imported severe acute respiratory syndrome coronavirus 2 cases by using importation predictions," Emerging Infectious Diseases, vol. 26, no. 7, pp. 1465-1469, 2020.

[14] JHCC, COVID-19, Vutfctwsa, https://coronavirus.jhu.edu/ data.

[15] R. Niehus, P. M. De Salazar, A. R. Taylor, and M. Lipsitch, "Using observational data to quantify bias of traveller-derived COVID-19 prevalence estimates in Wuhan, China," The Lancet Infectious Diseases, vol. 20, no. 7, pp. 803-808, 2020.

[16] M. Salathé, C. L. Althaus, R. Neher et al., "COVID-19 epidemic in Switzerland: on the importance of testing, contact tracing and isolation," Swiss Medical Weekly, vol. 150, no. 1112, p. w20225, 2020.

[17] D. Wang, B. Hu, C. Hu et al., "Clinical characteristics of 138 hospitalized patients with 2019 novel coronavirus-infected pneumonia in wuhan, China," Jama, vol. 323, no. 11, pp. 1061-1069, 2020.

[18] J. Yang, Y. Zheng, X. Gou et al., "Prevalence of comorbidities and its effects in patients infected with SARS-CoV-2: a systematic review and meta-analysis," International Journal of Infectious Diseases: Official Publication of the International Society for Infectious Diseases, vol. 94, pp. 91-95, 2020.

[19] P. K. Whelton, R. M. Carey, W. S. Aronow et al., "2017 ACC/ AHA/AAPA/ABC/ACPM/AGS/APhA/ASH/ASPC/NMA/ PCNA guideline for the prevention, detection, evaluation, and management of high blood pressure in adults: a report of the American College of Cardiology/American Heart Association 
Task Force on Clinical Pr," Journal of the American College of Cardiology, vol. 71, no. 19, pp. e127-e248, 2018.

[20] J. Cao, X. Hu, W. Cheng, L. Yu, W.-J. Tu, and Q. Liu, "Clinical features and short-term outcomes of 18 patients with corona virus disease 2019 in intensive care unit," Intensive Care Medicine, vol. 46, no. 5, pp. 851-853, 2020.

[21] C. Huang, Y. Wang, X. Li et al., "Clinical features of patients infected with 2019 novel coronavirus in Wuhan, China," The Lancet, vol. 395, no. 10223, pp. 497-506, 2020.

[22] C. Wu, X. Chen, Y. Cai et al., "Risk factors associated with acute respiratory distress syndrome and death in patients with coronavirus disease 2019 pneumonia in Wuhan, China," JAMA internal medicine, vol. 180, no. 7, pp. 934-943, 2020.

[23] W.-j. Guan, Z.-y. Ni, Y. Hu et al., "Clinical characteristics of coronavirus disease 2019 in China," New England Journal of Medicine, vol. 382, no. 18, pp. 1708-1720, 2020.

[24] C. P. E. R. E. Novel, "The epidemiological characteristics of an outbreak of 2019 novel coronavirus diseases (COVID-19) in China," Zhonghua Liu Xing Bing Xue Za Zhi = Zhonghua Liuxingbingxue Zazhi, vol. 41, no. 2, p. 145, 2020.

[25] J. Li, X. Wang, J. Chen, H. Zhang, and A. Deng, "Association of renin-angiotensin system inhibitors with severity or risk of death in patients with hypertension hospitalized for coronavirus disease 2019 (COVID-19) infection in Wuhan, China," JAMA cardiology, vol. 5, no. 7, pp. 825-830, 2020.

[26] J.-j. Zhang, X. Dong, Y.-y. Cao et al., "Clinical characteristics of 140 patients infected with SARS-CoV-2 in Wuhan, China," Allergy, vol. 75, no. 7, pp. 1730-1741, 2020.

[27] X. Cheng, G. Cai, X. Wen et al., "Clinical characteristics and fatal outcomes of hypertension in patients with severe COVID-19," Aging, vol. 12, no. 23, pp. 23436-23449, 2020.

[28] L. F. Drager, A. Pio-Abreu, R. D. Lopes, and L. A. Bortolotto, "Is hypertension a real risk factor for poor prognosis in the COVID-19 pandemic?" Current Hypertension Reports, vol. 22, no. 6, pp. 1-6, 2020.

[29] T.-Y. Xiong, F.-Y. Huang, Q. Liu et al., "Hypertension is a risk factor for adverse outcomes in patients with coronavirus disease 2019: a cohort study," Annals of Medicine, vol. 52, no. 7, pp. 361-366, 2020.

[30] R. Pranata, M. A. Lim, I. Huang, S. B Raharjo, and A. A Lukito, "Hypertension is associated with increased mortality and severity of disease in COVID-19 pneumonia: a systematic review, meta-analysis and meta-regression," Journal of the Renin-Angiotensin-Aldosterone System: Journal of the Renin-AngiotensinAldosterone System, vol. 21, no. 2, 2020.

[31] E. Rodilla, A. Saura, I. Jiménez et al., “Association of hypertension with all-cause mortality among hospitalized patients with COVID-19," Journal of Clinical Medicine, vol. 9, no. 10, p. $3136,2020$.

[32] X. Zhou, J. Zhu, and T. Xu, "Clinical characteristics of coronavirus disease 2019 (COVID-19) patients with hypertension on renin-angiotensin system inhibitors," Clinical \& Experimental Hypertension, vol. 42, no. 7, pp. 656-660, 2020.

[33] L. Roncon, M. Zuin, G. Zuliani, and G. Rigatelli, "Patients with arterial hypertension and COVID-19 are at higher risk of ICU admission," British Journal of Anaesthesia, vol. 125, no. 2, pp. e254-e255, 2020.

[34] R. C. W. Ma and R. I. G. Holt, "COVID-19 and diabetes," Diabetic Medicine, vol. 37, no. 5, pp. 723-725, 2020.

[35] E. L. Schiffrin, J. M. Flack, S. Ito, P. Muntner, and R. C. Webb, Hypertension and COVID-19, Oxford University Press, Oxford, UK, 2020.
[36] R. Pal and A. Bhansali, "COVID-19, diabetes mellitus and ACE2: the conundrum," Diabetes Research and Clinical Practice, vol. 162, p. 162, Article ID 108132, 2020.

[37] G. P. Rossi, V. Sanga, and M. Barton, "Potential harmful effects of discontinuing ACE-inhibitors and ARBs in COVID19 patients," Elife, vol. 9, Article ID e57278, 2020.

[38] L. Fang, G. Karakiulakis, and M. Roth, "Are patients with hypertension and diabetes mellitus at increased risk for COVID-19 infection?" The Lancet Respiratory Medicine, vol. 8, no. 4, p. e21, 2020.

[39] M. Hoffmann, H. Kleine-Weber, S. Schroeder et al., "SARSCoV-2 cell entry depends on ACE2 and TMPRSS2 and is blocked by a clinically proven protease inhibitor," Cell, vol. 181, no. 2, pp. 271-280, 2020.

[40] S. Huang, J. Wang, F. Liu et al., "COVID-19 patients with hypertension have more severe disease: a multicenter retrospective observational study," Hypertension Research, vol. 43, no. 8, pp. 824-831, 2020.

[41] D. Antwi-Amoabeng, B. D. Beutler, A. E. Moody, Z. Kanji, N. Gullapalli, and C. J. Rowan, "Management of hypertension in COVID-19," World Journal of Cardiology, vol. 12, no. 5, pp. 228-230, 2020.

[42] G. Lippi, J. Wong, and B. M. Henry, "Hypertension and its severity or mortality in coronavirus disease 2019 (COVID19): a pooled analysis," Polish Archives of Internal Medicine, vol. 130, no. 4, pp. 304-309, 2020.

[43] S. Kulkarni, B. L. Jenner, and I. Wilkinson, "COVID-19 and hypertension," Journal of the Renin-Angiotensin-Aldosterone System: Journal of the Renin-Angiotensin-Aldosterone System, vol. 21, no. 2, 2020.

[44] J.-C. Youn, H. T. Yu, B. J. Lim et al., "Immunosenescent CD8 $+\mathrm{T}$ cells and C-X-C chemokine receptor type 3 chemokines are increased in human hypertension," Hypertension, vol. 62, no. 1, pp. 126-133, 2013.

[45] S. Shi, M. Qin, B. Shen et al., "Association of cardiac injury with mortality in hospitalized patients with COVID-19 in Wuhan, China," JAMA cardiology, vol. 5, no. 7, pp. 802-810, 2020.

[46] S. Wang, Y. Chen, L. Wang, H. Liu, and P. Han, "Are COVID19 patients with hypertension at higher risk in China?" Journal of Hypertension, vol. 38, no. 7, pp. 1384-1385, 2020.

[47] D. Kifer, D. Bugada, J. Villar-Garcia et al., "Effects of environmental factors on severity and mortality of COVID-19," Frontiers of Medicine, vol. 7, Article ID 607786, 2020. 